\title{
A Note on Primes Between Consecutive Powers
}

\author{
Danilo BazZanella $(*)$
}

ABSTRACT - In this paper we carry on the study of the distribution of prime numbers between two consecutive powers of integers.

\section{Introduction.}

A well known conjecture about the distribution of primes asserts that all intervals of type $\left[n^{2},(n+1)^{2}\right]$ contain at least one prime. The proof of this conjecture is quite out of reach at present, even under the assumption of the Riemann Hypothesis. To get a conditional proof of the conjecture we need to assume a stronger hypothesis about the behaviour of Selberg's integral in short intervals, see D. Bazzanella [3]. This paper concerns with the distribution of prime numbers between two consecutive powers of integers, as a natural generalization of the above problem. The well known result of M. N. Huxley [8] about the distribution of prime in short intervals implies that all intervals $\left[n^{\alpha},(n+1)^{\alpha}\right]$ contain the expected number of primes for $\alpha>\frac{12}{5}$ and $n \rightarrow \infty$. This was slightly improved by D. R. HeathBrown [7] to $\alpha \geq \frac{12}{5}$.

Assuming some heuristic hypotheses we can obtain the expected distribution of primes for smaller values of $\alpha$. In particular under the assumption of the Lindelöf hypothesis, which states that the Riemann Zeta-function satisfies

$$
\zeta(\sigma+i t) \ll t^{\eta} \quad\left(\sigma \geq \frac{1}{2}, t \geq 2\right),
$$

(*) Indirizzo dell'A.: Dipartimento di Matematica, Politecnico di Torino, Corso Duca degli Abruzzi, 24, 10129 Torino, Italy.

E-mail: danilo.bazzanella@polito.it

1991 Mathematics Subject Classification. 11NO5. 
for any $\eta>0$, the classical result of A. E. Ingham [9] implies that all intervals $\left[n^{\alpha},(n+1)^{\alpha}\right]$ contain the expected number of primes for $\alpha>2$.

In a previous paper, see [2], the author proved that all intervals $\left[n^{\alpha},(n+1)^{\alpha}\right] \subset[N, 2 N]$, with at most $O(B(N, \alpha))$ exceptions, contain the expected number of primes, for suitable function $B(N, \alpha)$. More precisely the author proved that we can choose

$$
B(N, \alpha)= \begin{cases}\left(N^{1 / \alpha}\right)^{\frac{8}{5}-\frac{\alpha}{2}+\varepsilon} & \frac{6}{5}<\alpha \leq \frac{6}{5}+c \\ \left(N^{1 / \alpha}\right)^{\frac{5}{2}-\alpha+\varepsilon} & \frac{27}{16}<\alpha \leq \frac{53}{26} \\ \left(N^{1 / \alpha}\right)^{\frac{72-9 x-8 x^{2}}{3(\alpha+12)}+\varepsilon} & \frac{53}{26} \leq \alpha<\frac{12}{5}\end{cases}
$$

for $\varepsilon>0$ and $c$ a suitable positive constant. The author proved also that, under the assumption of the Lindelöf Hypothesis, we can choose

$$
B(N, \alpha)=\left(N^{1 / \alpha}\right)^{2-\alpha+\varepsilon} \text { for } 1<\alpha \leq 2
$$

and, under the assumption of the Riemann Hypothesis, we can choose

$$
B(N, \alpha)=\left(N^{1 / \alpha}\right)^{2-\alpha} \log ^{2} N g(N) \text { for } \quad 1<\alpha \leq 2,
$$

with $g(N) \rightarrow \infty$ arbitrarily slowly.

In this paper we establish the upper bounds for the exceptional set of the distribution of primes between two consecutive powers of integers under the assumption of some other heuristic hypotheses. The first hypothesis regards the counting functions $N(\sigma, T)$ and $N^{*}(\sigma, T)$. The former is defined as the number of zeros $\rho=\beta+i \gamma$ of Riemann zeta function which satisfy $\sigma \leq \beta \leq 1$ and $|\gamma| \leq T$, while $N^{*}(\sigma, T)$ is defined as the number of ordered sets of zeros $\rho_{j}=\beta_{j}+i \gamma_{j}$ $(1 \leq j \leq 4)$, each counted by $N(\sigma, T)$, for which $\left|\gamma_{1}+\gamma_{2}-\gamma_{3}-\gamma_{4}\right| \leq 1$. If we make the heuristic assumption that there exists a constant $T_{0}$ such that

$$
N^{*}(\sigma, T) \ll \frac{N(\sigma, T)^{4}}{T} \quad\left(\frac{1}{2} \leq \sigma \leq 1, T \geq T_{0}\right)
$$

as in D. Bazzanella and A. Perelli [4], then we can obtain the following result.

THeorem 1. Assume (4) and let $\varepsilon>0$. Then all intervals $\left[n^{\alpha},(n+1)^{\alpha}\right] \subset$ $\subset[N, 2 N]$, with at most $O\left(\left(N^{1 / \alpha}\right)^{\eta(\alpha)+\varepsilon}\right)$ exceptions, contain the expected 
number of primes, where

$$
\eta(\alpha)=\left\{\begin{array}{ll}
4 \alpha+12-8 \sqrt{3 \alpha} & \frac{27}{16} \leq \alpha \leq \frac{48}{25} \\
\frac{12}{5}-\alpha & \frac{48}{25} \leq \alpha<\frac{12}{5}
\end{array} .\right.
$$

For $\alpha$ near $6 / 5$ the assumption of (4) is not helpful to obtain a stronger result than the unconditional result (1) proved in [2]. A corollary of this theorem is Theorem 3 of D. Bazzanella [1], which states that, under the assumption of (4), all intervals $\left[n^{2},(n+1)^{2}\right] \subset[N, 2 N]$, with at most $O\left(N^{1 / 5+\varepsilon}\right)$ exceptions, contain the expected number of primes. Recalling that for $\alpha \geq 12 / 5$ there are not exceptions, we expect to have

$$
\lim _{\alpha \rightarrow 12 / 5^{-}} \eta(\alpha)=0 \text {. }
$$

We note that the above condition is implies by the Theorem 1 , but not by the unconditional result (1).

Moreover we assume the Density Hypothesis, which states that for every $\eta>0$ the counting function $N(\sigma, T)$ satisfies

$$
N(\sigma, T) \ll T^{2(1-\sigma)+\eta} \quad\left(\frac{1}{2} \leq \sigma \leq 1\right),
$$

obtaining our last result.

THEOREM 2. Assume the Density Hypothesis and (4), let $\varepsilon>0$ and $1<\alpha \leq 2$. Then all intervals $\left[n^{\alpha},(n+1)^{\alpha}\right] \subset[N, 2 N]$, with at most $O\left(\left(N^{1 / \alpha}\right)^{\eta(\alpha)+\varepsilon}\right)$ exceptions, contain the expected number of primes, where

$$
\eta(\alpha)=2(2-\alpha)
$$

If we assume the Riemann Hypothesis, it is known that for $\alpha>2$ there are not exceptions and then we expect to have $\eta(2)=0$. Indeed, although the assumptions of the Theorem 2 are weaker than the Riemann Hypothesis, we obtain $\eta(2)=0$ again.

\section{The basic lemma.}

Throughout the paper we always assume that $n, x, X$ and $N$ are sufficiently large as prescribed by the various statements, and $\varepsilon>0$ is arbitrarily small and not necessary the same at each occurrence. The basic 
lemma is a result about the structure of the exceptional set for the asymptotic formula

$$
\psi(x+h(x))-\psi(x) \sim h(x) \quad \text { as } \quad x \rightarrow \infty .
$$

Let || denote the modulus of a complex number or the Lebesgue measure of an infinite set of real numbers or the cardinality of a finite set. Let $\delta>0$ and let $h(x)$ be an increasing function such that $x^{\varepsilon} \leq h(x) \leq x$ for some $\varepsilon>0$,

$$
\Delta(x, h)=\psi(x+h(x))-\psi(x)-h(x)
$$

and

$$
E_{\delta}(X, h)=\{X \leq x \leq 2 X:|\Delta(x, h)| \geq \delta h(x)\} .
$$

It is clear that (5) holds if and only if for every $\delta>0$ there exists $X_{0}(\delta)$ such that $E_{\delta}(X, h)=\emptyset$ for $X \geq X_{0}(\delta)$. Hence for small $\delta>0, X$ tending to $\infty$ and $h(x)$ suitably small with respect to $x$, the set $E_{\delta}(X, h)$ contains the exceptions, if any, to the expected asymptotic formula for the number of primes in short intervals. Moreover, we observe that

$$
E_{\delta}(X, h) \subset E_{\delta^{\prime}}(X, h) \quad \text { if } \quad 0<\delta^{\prime}<\delta .
$$

We will consider increasing functions $h(x)$ of the form $h(x)=x^{\theta+\varepsilon(x)}$, with some $0<\theta<1$ and a function $\varepsilon(x)$ such that $|\varepsilon(x)|$ is decreasing,

$$
\varepsilon(x)=o(1) \quad \text { and } \quad \varepsilon(x+y)=\varepsilon(x)+O\left(\frac{|y|}{x \log x}\right),
$$

for every $|y|<x$. A function satisfying these requirements will be called of type $\theta$.

The basic lemma provides the structure of the exceptional set $E_{\delta}(X, h)$.

Lemma. Let $0<\theta<1, h(x)$ be of type $\theta, X$ be sufficiently large depending on the function $h(x)$ and $0<\delta^{\prime}<\delta$ with $\delta-\delta^{\prime} \geq \exp (-\sqrt{\log X})$. If $x_{0} \in E_{\delta}(X, h)$ then $E_{\delta^{\prime}}(X, h)$ contains the interval $\left[x_{0}-\operatorname{ch}(X)\right.$, $\left.x_{0}+\operatorname{ch}(X)\right] \cap[X, 2 X]$, where $c=\left(\delta-\delta^{\prime}\right) \theta / 5$. In particular, if $E_{\delta}(X, h) \neq \emptyset$ then

$$
\left|E_{\delta^{\prime}}(X, h)\right| \gg_{\theta}\left(\delta-\delta^{\prime}\right) h(X) .
$$

The above Lemma is part (i) of Theorem 1 of D. Bazzanella and A. Perelli, see [4], and it essentially says that if we have a single exception in $E_{\delta}(X, h)$, with a fixed $\delta$, then we necessarily have an interval of exceptions in $E_{\delta^{\prime}}(X, h)$, with $\delta^{\prime}$ a little smaller than $\delta$. 


\section{Proof of the Theorems.}

We define $H=(n+1)^{\alpha}-n^{\alpha}$ and

$$
A_{\delta}(N, \alpha)=\left\{N^{1 / \alpha} \leq n \leq(2 N)^{1 / \alpha}:\left|\psi\left((n+1)^{\alpha}\right)-\psi\left(n^{\alpha}\right)-H\right| \geq \delta H\right\} .
$$

This set contains the exceptions, if any, to the expected asymptotic formula for the number of primes in intervals of the type $\left[n^{\alpha},(n+1)^{\alpha}\right] \subset[N, 2 N]$.

The main step of the proof is to connect the exceptional set $A_{\delta}(N, \alpha)$ with the exceptional set for the distribution of primes in short intervals and to show that

$$
\left|A_{\delta}(N, \alpha)\right| \ll \frac{\left|E_{\delta / 2}(N, h)\right|}{N^{1-1 / \alpha}}+1,
$$

for every $\delta>0, \alpha>1$ and $h(x)=\left(x^{1 / \alpha}+1\right)^{\alpha}-x$.

In order to prove (6) we choose $n \in A_{\delta}(N, \alpha)$ and let $x=n^{\alpha} \in[N, 2 N]$. From the definition of $A_{\delta}(N, \alpha)$ we get

$$
\left|\psi\left((n+1)^{\alpha}\right)-\psi\left(n^{\alpha}\right)-H\right| \geq \delta H,
$$

and then

$$
|\psi(x+h(x))-\psi(x)-h(x)| \geq \delta h(x),
$$

which implies that $x \in E_{\delta}(N, h)$. Using the Lemma, with $\delta^{\prime}=\delta / 2$, we obtain that there exists an effective constant $c$ such that

$$
[x, x+\operatorname{ch}(x)] \cap[N, 2 N] \subset E_{\delta / 2}(N, h) .
$$

Let $m \in A_{\delta}(N, \alpha), m>n$. As before we can define $y=m^{\alpha} \in[N, 2 N]$ such that

$$
[y, y+\operatorname{ch}(y)] \cap[N, 2 N] \subset E_{\delta / 2}(N, h) .
$$

Choosing $c<1$ we find

$$
y-x=m^{\alpha}-n^{\alpha} \geq(n+1)^{\alpha}-n^{\alpha}>\operatorname{ch}(x),
$$

and then

$$
[x, x+\operatorname{ch}(x)] \cap[y, y+\operatorname{ch}(y)]=\emptyset .
$$

Hence (6) is proved, since for every $n \in A_{\delta}(N, \alpha)$ and $x=n^{\alpha}$, with at most one exception, we have

$$
[x, x+\operatorname{ch}(x)] \subset[N, 2 N] .
$$

Now we can conclude the proof of the theorems providing a suitable bounds for the measure of the exceptional set $E_{\delta / 2}(N, h)$. 
If we consider $x \in E_{\delta / 2}(N, h)$ we get

$$
|\psi(x+h(x))-\psi(x)-h(x)| \gg N^{1-1 / \alpha}
$$

and then

$$
\begin{gathered}
\left|E_{\delta / 2}(N, h)\right| N^{4-4 / \alpha} \\
\ll \int_{E_{\delta / 2}(N, h)}|\psi(x+h(x))-\psi(x)-h(x)|^{4} \mathrm{~d} x \\
\ll \int_{N}^{2 N}|\psi(x+h(x))-\psi(x)-h(x)+\Sigma(x)|^{4} \mathrm{~d} x,
\end{gathered}
$$

for every $\Sigma(x)$ such that

$$
\Sigma(x) \ll \frac{N^{1-1 / \alpha}}{\log N} .
$$

Now we use the classical explicit formula, see H. Davenport [5, Chapter 17], to write

$$
\psi(x+h(x))-\psi(x)-h(x)=-\sum_{|\gamma| \leq T} x^{\rho} c_{\rho}(x)+O\left(\frac{N \log ^{2} N}{T}\right),
$$

uniformly for $N \leq x \leq 2 N$, where $10 \leq T \leq N, \rho=\beta+i \gamma$ runs over the non-trivial zeros of $\zeta(s)$ and

$$
c_{\rho}(x)=\frac{(1+h(x) / x)^{\rho}-1}{\rho} .
$$

Let

$$
T=N^{1 / \alpha} \log ^{3} N
$$

and then

$$
c_{\rho}(x) \ll \min \left(N^{-1 / \alpha}, \frac{1}{|\gamma|}\right) .
$$

Follow the method of D. R. Heath-Brown, see [6], we find a constant $0<u<1$ such that

$$
\sum_{|\gamma| \leq T, \beta>u} x^{\rho} c_{\rho}(x) \ll \frac{N^{1-1 / \alpha}}{\log N}
$$


obtaining

$$
\psi(x+h(x))-\psi(x)-h(x)=-\sum_{|\gamma| \leq T, \beta \leq u} x^{\rho} c_{\rho}(x)+O\left(\frac{N^{1-1 / \alpha}}{\log N}\right),
$$

and then, from (7), we have

$$
\left|E_{\delta}(N, h)\right| N^{4-4 / \alpha} \ll \int_{N}^{2 N}\left|\sum_{|\gamma| \leq T, \beta \leq u} x^{\rho} c_{\rho}(x)\right|^{4} \mathrm{~d} x .
$$

To estimate the fourth power integral we divide the interval $[0, u]$ into $O(\ln N)$ subintervals $I_{k}$ of the form

$$
I_{k}=\left[\frac{k}{\log N}, \frac{k+1}{\log N}\right]
$$

and by Hölder inequality we obtain

$$
\left|\sum_{|\gamma| \leq T, \beta \leq u} x^{\rho} c_{\rho}(x)\right|^{4} \ll \ln ^{3} N \sum_{k}\left|\sum_{|\gamma| \leq T, \beta \in I_{k}} x^{\rho} c_{\rho}(x)\right|^{4}
$$

and then

$$
\int_{N}^{2 N}\left|\sum_{|\gamma| \leq T, \beta \leq u} x^{\rho} c_{\rho}(x)\right|^{4} d x \ll N^{1-4 / \alpha+\varepsilon} \max _{\sigma \leq u} N^{4 \sigma} M(\sigma, T),
$$

where

$$
M(\sigma, T)=\sum_{\substack{\beta_{1}, \ldots, \beta_{4} \geq \sigma \\\left|\gamma_{1}\right| \leq T, \ldots, \gamma_{4} \mid \leq T}} \frac{1}{1+\left|\gamma_{1}+\gamma_{2}-\gamma_{3}-\gamma_{4}\right|}
$$

It is not difficult to prove that

$$
M(\sigma, T) \ll N^{*}(\sigma, T) \log N,
$$

see [6], and then from (11) and (12) this yields

$$
\left|E_{\delta / 2}(N, h)\right| \ll N^{-3+\varepsilon} \max _{\sigma \leq u} N^{4 \sigma} N^{*}(\sigma, T) .
$$

The assumption of (4) then implies

$$
\left|E_{\delta / 2}(N, h)\right| \ll N^{-3-1 / \alpha+\varepsilon}\left(\max _{\sigma \leq u} N^{\sigma} N(\sigma, T)\right)^{4} .
$$


Using the Ingham-Huxley density estimate, asserting that for every $v>0$ we have

$$
N(\sigma, T) \ll\left\{\begin{array}{ll}
T^{3(1-\sigma) /(2-\sigma)+v} & \frac{1}{2} \leq \sigma \leq \frac{3}{4} \\
T^{3(1-\sigma) /(3 \sigma-1)+v} & \frac{3}{4} \leq \sigma \leq 1
\end{array},\right.
$$

see [10, Theorem 11.1], we obtain an upper bound that for $\alpha \geq 48 / 25$ attains its maximum at $\sigma=3 / 4$, and so we get

$$
\left|E_{\delta / 2}(N, h)\right| \ll N^{-3-1 / \alpha+\varepsilon} N^{4}\left(3 / 4+\frac{3}{5 \alpha}\right) \ll N^{\frac{7}{5 \alpha}}+\varepsilon .
$$

From (6) and (15) we can conclude

$$
\begin{gathered}
\left|A_{\delta}(N, \alpha)\right| \ll \frac{\left|E_{\delta / 2}(N, h)\right|}{N^{1-1 / \alpha}}+1 \ll \frac{N^{5 \alpha}+\varepsilon}{N^{1-1 / \alpha}} \\
\ll N^{12}-1+\varepsilon_{\ll} \ll\left(N^{1 / \alpha}\right)\left(\frac{12}{5}-\alpha\right)+\varepsilon,
\end{gathered}
$$

for every $\delta>0$ and $\alpha \geq 48 / 25$.

For $27 / 16 \leq \alpha \leq 48 / 25$ the above bound attains its maximum at $\bar{\sigma}=2-\sqrt{3 / \alpha}$ and then we have

$$
\left|E_{\delta / 2}(N, h)\right| \ll N^{-3-1 / \alpha+\varepsilon}\left(N^{\bar{\sigma}} N(\bar{\sigma}, T)\right)^{4} \ll N^{5+\frac{11}{\alpha}-8 \sqrt{3 / \alpha}+\varepsilon} .
$$

Thus, from (6) and (16), we deduce

$$
\begin{gathered}
\left|A_{\delta}(N, \alpha)\right| \ll \frac{\left|E_{\delta / 2}(N, h)\right|}{N^{1-1 / \alpha}}+1 \ll \frac{N^{5+\frac{11}{\alpha}-8 \sqrt{3 / \alpha}+\varepsilon}}{N^{1-1 / \alpha}} \\
\ll N^{4+\frac{12}{\alpha}-8 \sqrt{3 / \alpha+\varepsilon}} \ll\left(N^{1 / \alpha}\right)^{(4 \alpha+12-8 \sqrt{3 \alpha})+\varepsilon} .
\end{gathered}
$$

for every $\delta>0$ and $27 / 16 \leq \alpha \leq 48 / 25$, and then Theorem 1 follows.

In order to prove Theorem 2 we imitate the proof of Theorem 1 up to equation (13) and then we write

$$
\left|E_{\delta / 2}(N, h)\right| \ll N^{-3-1 / \alpha+\varepsilon}\left(\max _{\sigma \leq u} N^{\sigma} N(\sigma, T)\right)^{4} .
$$

Recalling that under the assumption of the Density Hypothesis we have

$$
N(\sigma, T) \ll \begin{cases}T^{2(1-\sigma)+v} & \frac{1}{2} \leq \sigma \leq \frac{11}{14} \\ T^{9(1-\sigma) /(7 \sigma-1)+v} & \frac{11}{14} \leq \sigma \leq 1\end{cases}
$$


for every $v>0$, we thus obtain an upper bound for the exceptional set. For every $1<\alpha \leq 2$ such a bound attains its maximum at $\sigma=1 / 2$, and so we obtain

$$
\left|E_{\delta / 2}(N, h)\right| \ll N^{-3-1 / \alpha+\varepsilon}\left(N^{2} N(1 / 2, T)\right)^{4} \ll N^{3 / \alpha-1+\varepsilon}
$$

The above bound and (6) imply that

$$
\begin{gathered}
\left|A_{\delta}(N, \alpha)\right| \ll \frac{\left|E_{\delta / 2}(N, h)\right|}{N^{1-1 / \alpha}}+1 \ll \frac{N^{3 / \alpha-1+\varepsilon}}{N^{1-1 / \alpha}} \\
\ll N^{4 / \alpha-2+\varepsilon} \ll\left(N^{1 / \alpha}\right)^{(4-2 \alpha)+\varepsilon} .
\end{gathered}
$$

This concludes the proof of Theorem 2 .

\section{REFERENCES}

[1] D. Bazzanella, The exceptional set for the distribution of primes between consecutive squares, Preprint 2008.

[2] D. Bazzanella, The exceptional set for the distribution of primes between consecutive powers, Acta Math. Hungar, 116 (3) (2007), pp. 197-207.

[3] D. Bazzanella, Primes between consecutive square, Arch. Math., 75 (2000), pp. 29-34.

[4] D. Bazzanella - A. Perelli, The exceptional set for the number of primes in short intervals, J. Number Theory, 80 (2000), pp. 109-124 .

[5] H. DavenPoRT, Multiplicative Number Theory, volume GTM 74 (Springer Verlag, 1980), second edition.

[6] D. R. HeAth-Brown, The difference between consecutive primes II, J. London Math. Soc., 19 (2) (1979), pp. 207-220.

[7] D. R. Heath-Brown, The number of primes in a short interval. J. Reine Angew. Math., 389 (1988), pp. 22-63.

[8] M. N. HuXLeY, On the difference between consecutive primes, Invent. Math., 15 (1972), pp. 164-170.

[9] A. E. InGHAm, On the difference between consecutive primes, Quart. J. of Math. (Oxford), 8 (1937), pp. 255-266.

[10] A. Ivić, The Riemann Zeta-Function, John Wiley and Sons, New York, 1985.

Manoscritto pervenuto in redazione l'11 febbraio 2008. 
